\title{
Comparação da função visual e do índice de qualidade de vida com uso de óculos ou de lente de contato progressiva
}

\author{
Comparative analysis of visual function and the quality of life index with \\ eyeglasses or a progressive contact lens
}

\author{
Neusa Vidal Sant'Anna ${ }^{1}$ \\ Paulo Schor $^{2}$ \\ César Lipener ${ }^{3}$ \\ Ricardo Uras ${ }^{4}$
}

Trabalho realizado no Departamento de Oftalmologia da Universidade Federal de São Paulo - UNIFESP.

${ }^{1}$ Oftalmologista pós-graduanda nível doutorado pela Universidade Federal de São Paulo - UNIFESP - São Paulo (SP) - Brasil.

${ }^{2}$ Doutor livre-docente e Chefe do setor de Bioengenharia da UNIFESP - São Paulo (SP) - Brasil.

${ }^{3}$ Chefe do Setor de Lente de Contato e Refração da UNIFESP - São Paulo (SP) - Brasil.

${ }^{4}$ Professor Adjunto do Departamento de Oftalmologia da UNIFESP - São Paulo (SP) - Brasil.

Endereco para correspondência: Neusa Vidal Sant'Anna. Rua Voluntários da Pátria, 1916 - Sala 4 - São Paulo (SP) CEP 02010-600

E-mail: neusa.vidal@bol.com.br

Recebido para publicação em 13.10.2004

Versão revisada recebida em 12.01.2006

Aprovação em 31.01.2006

O presente trabalho foi realizado sem patrocínio e apresenta dados parciais da tese de doutorado da Dra. Neusa Vidal, defendida em 2004 na UNIFESP. As lentes de contato do estudo foram doadas pela Ciba Vision.

Nota Editorial: Depois de concluída a análise do artigo sob sigilo editorial e com a anuência da Dra. Edméa Rita Temporini sobre a divulgação de seu nome como revisora, agradecemos sua participação neste processo.

\section{RESUMO}

Objetivo: Comparar a função visual e a resposta dos pacientes ao índice de qualidade de vida quando estes são corrigidos com óculos com lentes progressivas ou lente de contato progressiva. Métodos: Foram selecionados 35 pacientes présbitas, usuários de óculos com acuidade visual igual, ou melhor, a logMAR zero (longe) e J1 (perto), para adaptarem a lente de contato Focus Progressive ${ }^{\circledR}$. Foram comparadas medidas de acuidade visual para longe, perto e sensibilidade ao contraste com a lente de contato e com os óculos. Os resultados do questionário de avaliação de qualidade de vida NEI VFQ-25 dos mesmos pacientes corrigidos com os óculos e com a lente de contato progressiva foram comparados, levando-se em consideração o tipo de ametropia e a idade. Resultados: A acuidade visual para longe, perto e a sensibilidade ao contraste foram significantemente piores com lente de contato progressiva do que com os óculos. As respostas do questionário não diferiram quanto à forma de correção quando se analisou o tipo de ametropia, entretanto foram significantemente piores com a lente de contato nos grupos míope e hipermétrope abaixo das suas respectivas medianas da idade e maiores no hipermétrope acima de sua mediana. Conclusões: As funções visuais foram significantemente piores com a lente de contato e o tipo de ametropia não influenciou nas respostas ao índice de qualidade de vida quanto à forma de correção, mas sim, quanto à idade.

Descritores: Presbiopia; Qualidade de vida; Acomodação ocular; Lentes de contato; Óculos; Acuidade visual; Questionários; Estudo comparativo

\section{INTRODUÇ̃̃O}

A presbiopia é um processo fisiológico universal, imutável e progressivo com o envelhecimento ocular. É freqüente em indivíduos com mais de 40 anos de idade no Brasil, tendo seu início em diferentes faixas etárias pelo mundo. Seu aspecto evolutivo faz com que a acuidade visual para perto se deteriore gradativamente e o uso de uma correção óptica se torna imprescindível $^{(1)}$. Essa pode ser feita com óculos ou com lentes de contato. Os óculos podem ser monofocais, bifocais, trifocais ou progressivos, apresentando vantagens e desvantagens.

Os monofocais para perto proporcionam excelente acuidade visual e campo de visão, enquanto a amplitude de acomodação ainda é suficiente para suprir a visão para meia-distância. Os bifocais apresentam desempe- 
nhos ópticos de longe e de perto semelhantes aos respectivos óculos monofocais e campo de visão satisfatório; entretanto, suas desvantagens decorrem dos efeitos prismáticos, do efeito antiestético e da falta de correção para a visão intermediária ${ }^{(2)}$.

Os trifocais possuem uma zona óptica para longe, uma para distância intermediária e outra para perto, sendo que a de distância intermediária tem metade do valor em dioptrias da adição de perto e duas linhas que induzem salto de imagem. A impopularidade dessas lentes existe em decorrência da limitação do poder da zona da visão de distância intermediária, o que pode não satisfazer as necessidades do paciente présbita mais idoso, onde a acomodação é menor; da necessidade de uma montagem cuidadosa; do salto de imagem e do fator antiestético ${ }^{(3)}$.

Os óculos com lentes progressivas permitem visão para longe, perto e distância intermediária, por meio de mudanças na curvatura da superfície anterior, gerando poderes dióptricos progressivamente diferentes. Isso faz com que a lente tenha um aumento progressivo de sua positividade da área de longe em direção à área de perto. Possuem como desvantagens uma maior quantidade de aberrações quando se olha fora dos centros ópticos de longe e de perto, e um menor campo de visão ${ }^{(3)}$.

As aberrações inerentes a todos os óculos com lentes progressivas, a necessidade de uma montagem cuidadosa e a nova postura para procurar a posição adequada para a distância de leitura fazem com que muitos não se adaptem aos óculos com lentes progressivas, conforme alguns autores demonstraram avaliando 80 pacientes présbitas usuários de duas marcas diferentes de lentes progressivas ${ }^{(4)}$.

O interesse de oftalmologistas e fabricantes de lentes de contato por novas formas de correção óptica para a presbiopia cresce em função de problemas estéticos e ópticos inerentes aos óculos, somados ao aumento de uma população présbita, usuária previamente de lentes de contato e desejosa de outra forma de correção óptica para a presbiopia, que não os óculos ${ }^{(5)}$.

Estudos têm demonstrado um sucesso limitado com essas lentes disponíveis no mercado, apesar da acuidade visual ser satisfatória para perto, longe, distância intermediária e não existirem alterações significantes na sensibilidade ao contraste ${ }^{(6-9)}$.

Os valores discrepantes das avaliações subjetivas quando comparadas com os dados objetivos de desempenho óptico dessas lentes ocorrem em função da percepção visual do paciente ser dinâmica e levar em consideração muitos outros fatores não mensuráveis com as ferramentas objetivas atualmente disponíveis ${ }^{(6)}$. Desta forma, o surgimento de instrumentos que avaliem tanto a qualidade de vida como a função visual, têm se mostrado útil na avaliação e comparação de diversas formas de correções ópticas ${ }^{(10-12)}$.

Dois questionários específicos relacionados à visão foram desenvolvidos com a finalidade de avaliar tratamentos oculares: o "Visual functioning index" e o "National Eye Institute Visual Function Questionnaire-NEI-VFQ", cuja validade e confiabilidade têm sido mostrada em populações diferentes ${ }^{(13)}$.

Destes, o primeiro é usado para avaliar tratamentos de pacientes com catarata e o NEI-VFQ foi desenvolvido para avaliar qualidade de vida de um amplo aspecto de condições oculares $^{(11-14)}$, estando validado e disponível na língua portu$\operatorname{guesa}^{(11)}$.

Os objetivos deste estudo foram: a) comparar as medidas de acuidade visual para longe, perto e a sensibilidade ao contraste em pacientes présbitas corrigidos com os óculos com lentes progressivas ou com lente de contato; b) comparar as respostas do questionário de qualidade de vida NEI VFQ-25 quando os pacientes usavam óculos ou a lente de contato, levando-se em consideração os fatores idade e tipo de ametropia.

\section{MÉTODOS}

Esse estudo clínico prospectivo foi desenvolvido no Setor de Lente de contato e Refração do Departamento de Oftalmologia da Universidade Federal de São Paulo/Escola Paulista de Medicina (UNIFESP/EPM), de agosto a dezembro de 2003 após o protocolo ter sido analisado e aprovado pelo Comitê de Ética Médica do Hospital São Paulo (HSP) - UNIFESP, em junho de 2003.

Foram selecionados, pacientes présbitas, com mais de 40 anos, motivados a testar uma lente de contato progressiva, disponível no mercado comercialmente com o nome de Focus Progressive $^{\circledast}$, que atenderam ao anúncio publicado em cartazes afixados no Hospital São Paulo.

Os pacientes foram submetidos a uma avaliação inicial que incluía refração, medida da acuidade visual para longe na tabela parametrizada de Bailey-Lovie a $4 \mathrm{~m}$, para perto na carta de Jaeger, sensibilidade ao contraste na tabela VCTS 6500 a $3 \mathrm{~m}$, tonometria de aplanação, biomocroscopia, fundoscopia e ceratometria.

Foram selecionados pacientes emétropes, míopes, hipermétropes e astigmatas com astigmatismo composto, cujo componente cilíndrico fosse menor do que $1,00 \mathrm{D}$ e até $1 / 3$ do valor do componente esférico, com acuidade visual para longe zero $\operatorname{logMAR}$ e J1 para perto e sem doenças que contra-indicassem o uso de lentes de contato.

Prescreviam-se os óculos com lentes progressivas e após quatro semanas de uso, os pacientes respondiam o questionário NEI VFQ-25, o qual era composto de 25 perguntas agrupadas em 12 subdomínios, onde cada subdomínio possuía uma ou mais questões. Os subdomínios eram "saúde em geral", "visão em geral", "dor ocular", "atividades de perto", "atividades de longe", "aspectos sociais", "saúde mental", "atividades da vida diária", "dependência", "capacidade de dirigir", "visão de cores" e "visão periférica". Os pacientes foram orientados a responder às questões do questionário, levando em consideração a correção visual que usavam no momento. As alternativas escolhidas eram pontuadas de 0 a 100 de acordo com as normas propostas pelos autores do questionário. O escore de cada subdomínio era calculado pela média aritmética das questões que o compunham. Um escore total era ainda determinado, calculando-se a média aritmética dos 
12 subdomínios. Tanto os escores dos subdomínios como o escore total podiam variar de 0 a 100 , sendo que escores maiores indicavam melhor qualidade de vida.

Qualidade de vida foi definida como a medida da capacidade funcional do indivíduo em um aspecto multidimensional com sua saúde física, emocional, habilidade funcional e social, levando em consideração a sua opinião subjetiva quanto ao conceito de qualidade ${ }^{(12)}$.

Posteriormente, eram realizados os testes com a lente de contato Focus Progressive ${ }^{\circledR}$ (lente de contato progressiva, descartável de desenho asférico anterior, com 55\% de água e curvas base 8,6 ou $8,9 \mathrm{~mm}$ e de poder dióptrico $+6,00$ a -7,00D), onde o poder da lente era calculado segundo orientação do fabricante, dividindo-se o equivalente esférico da refração de longe por metade da adição e a curva base era escolhida de acordo com as medidas ceratométricas. Após a adaptação das lentes, aguardava-se 30 minutos para a avaliação biomicroscópica e sobre-refração. As lentes eram solicitadas ao fabricante, sem ônus aos pacientes. Quando chegavam, era medida a acuidade visual para longe, perto, a sensibilidade ao contraste e feita a avaliação biomicroscópica. Após a liberação das lentes de contato, os pacientes recebiam orientações quanto ao sistema de limpeza e manutenção das lentes e retornavam com quatro semanas de uso das lentes para reavaliar a acuidade visual de longe, perto, a sensibilidade ao contraste e responderem ao questionário NEI VFQ-25, considerando desta vez o uso das lentes de contato.

Foram analisadas as respostas do questionário considerando-se o escore total assim como os escores de cada subdomínio, levando-se em consideração o tipo de ametropia e a idade. Para tal, os pacientes foram agrupados em portadores de ametropias miópicas (miopia ou astigmatismo miópico composto) e ametropias hipermetrópicas (hipermétrope ou astigmatismo hipermetrópico composto) e a mediana da idade de cada grupo foi calculada.O teste de Wilcoxon foi utilizado para comparar o escore total, assim como os subdomínios destes pacientes corrigidos com os óculos ou com a lente de contato.

\section{RESULTADOS}

Dos 45 pacientes arrolados inicialmente neste estudo, dez foram excluídos ( 6 por possuírem astigmatismo simples e 4 por não atingirem acuidade visual para longe igual ou superior a zero $\log$ MAR). Dos 35 pacientes incluídos, cinco pacientes eram do sexo masculino $(14,3 \%)$ e 30 do sexo feminino $(85,7 \%)$

Quanto à profissão: 32 eram médicos, dois eram cirurgiões dentistas e um era engenheiro civil.

De acordo com a ametropia, 10 tinham ametropias miópicas $(28,6 \%)$ e 25 tinham ametropias hipermetrópicas $(71,4 \%)$ e todos os pacientes usavam previamente óculos com lentes progressivas.

A idade variou de 40 a 62 anos (média de 50,3 anos). A mediana da idade no grupo hipermétrope foi 50 anos e do grupo míope 47 anos.

A ceratometria variou de 42,00 a $45,50 \mathrm{D}$, com média de
43,25D. O equivalente esférico variou de $-1,00$ a $-6,00 \mathrm{D}$ para as ametropias miópicas e de $+0,25$ a $+5,00 \mathrm{D}$ para as ametropias hipermetrópicas.

Considerando a adição: 15 pacientes $(42,9 \%)$ usavam entre $1,00 \mathrm{D}$ e $1,75 \mathrm{D}$ e $20(57,1 \%)$ usavam entre 2,00 e 2,50D; sendo que no grupo com ametropia hipermetrópica, nove usavam adição entre 1,00D e 1,75D (36\%) e 16 entre 2,00D e 2,50D (64\%).

Quanto ao grupo de 10 présbitas com ametropia miópica, seis usavam adição entre 1,00D e 1,75D (60\%) e quatro usavam entre 2,00D e 2,50D (40\%) (Tabela 1).

Todos os pacientes tinham acuidade visual binocular medida na tabela Bailey-Lovie com óculos de 0,00 e perto J1.

Quanto à acuidade visual com as lentes de contato, 20 pacientes $(57,1 \%)$ tiveram acuidade visual binocular medida na tabela $\log$ MAR $0,00,13(37,10 \%)$ tiveram 0,1 e $2(5,8 \%)$ tiveram 0,2 (Figura 1). Analisando-se a diferença de resultados obtidos na tabela Bailey-Lovie com óculos e com as lentes, por meio do teste de Wilcoxon, a acuidade visual para longe com os óculos foi significantemente melhor do que com as lentes de contato $(\mathrm{p}<0,05)$.

Todos os pacientes apresentaram acuidade visual com óculos para perto $=\mathrm{J} 1,26$ pacientes com a lente de contato $(74,28 \%)$ atingiram J1 e nove pacientes $(25,72 \%)$ atingiram J2. Comparando-se a acuidade visual para perto segundo a forma de correção óptica, a acuidade visual com os óculos foi significantemente melhor do que com as lentes de contato $(p<0,05)$.

A média da sensibilidade ao contraste dos 35 pacientes présbitas com os óculos, medida binocularmente, foi igual à

\begin{tabular}{|c|}
\hline $\begin{array}{c}\text { Tabela 1. Distribuição da amostra de } 35 \text { pacientes présbitas de } \\
\text { acordo com adição }\end{array}$ \\
\begin{tabular}{cc} 
Adição & $\mathbf{N}(\%)$ \\
$+1,00 \mathrm{D}$ a $+1,75 \mathrm{D}$ & $15(42,9 \%)$ \\
$+2,00 \mathrm{D}$ a $+2,50 \mathrm{D}$ & $20(57,1 \%)$ \\
Total & $35(100 \%)$ \\
\hline
\end{tabular}
\end{tabular}

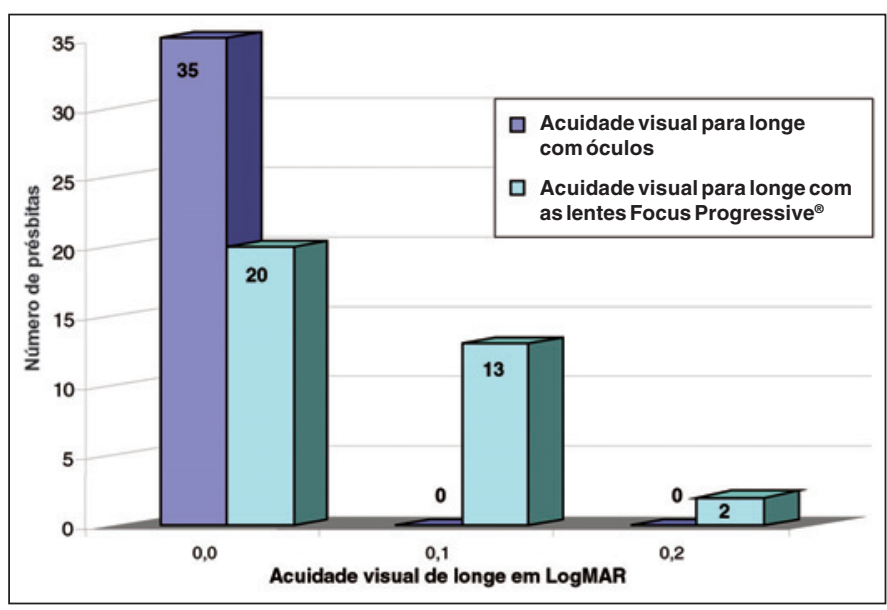

Figura 1 - Acuidade visual binocular de longe na tabela Bailey-Lovie com óculos e com as lentes Focus Progressive ${ }^{\circledR}$ após 4 semanas de uso das correções ópticas 


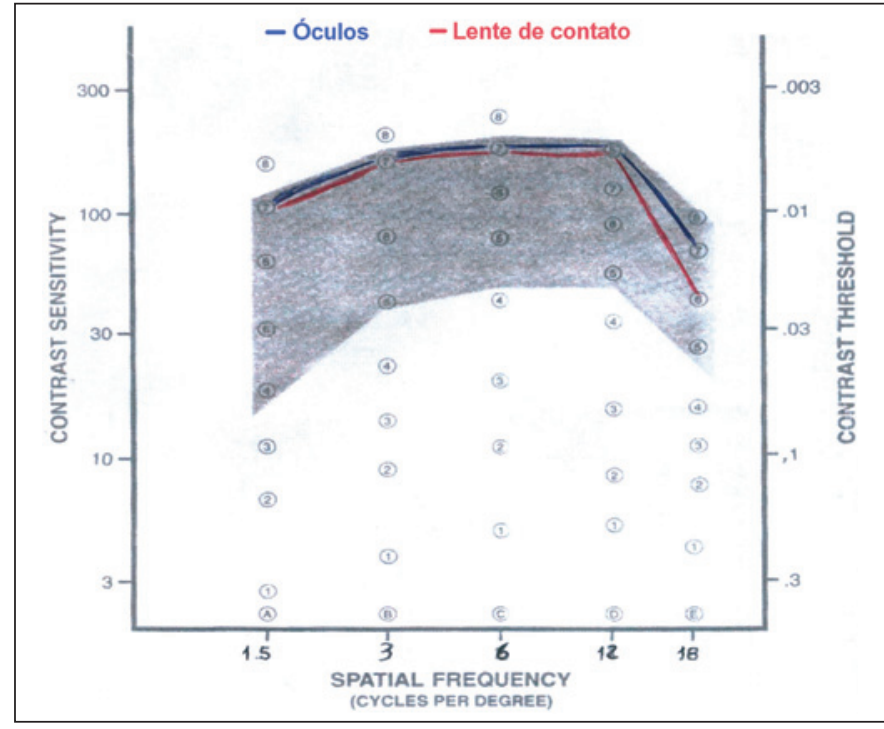

Figura 2 - Distribuição da média da sensibilidade ao contraste medida a $3 \mathrm{~m}$ na tabela VCTS 6500 binocularmente, nos 35 pacientes présbitas usando os óculos ou a lente de contato Focus Progressive ${ }^{\circledR}$ medida com a lente Focus Progressive ${ }^{\circledR}$ nas freqüências espaciais de 1,5 a 12 ciclos/grau, sendo significantemente menor com a lente de contato na freqüência de 18 ciclos/grau $(\mathrm{p}<0,01)$, porém, ainda situada dentro da área normal do gráfico de sensibilidade ao contraste (Figura 2).

$\mathrm{Na}$ análise dos resultados do questionário NEI VFQ-25, a média do escore total alcançado pela amostra de 35 présbitas quando em uso de óculos foi 84,43 e com a lente de contato Focus Progressive ${ }^{\circledR}$ foi 83,63 . Essa diferença analisada pelo teste de Wilcoxon não foi significante $(\mathrm{p}>0,05)$. Analisando-se, porém os subdomínios, observou-se que o subdomínio relativo à visão obteve escore significantemente maior quando estes pacientes estavam usando os óculos ( $\mathrm{p}<0,05)$ (Tabelas 2, 3 e 4).

Analisando-se os resultados do questionário dos 25 pacientes com ametropia hipermetrópica quando em uso dos óculos ou da lente de contato, notou-se que a média do escore total com o uso de óculos foi 84,15 e com a lente Focus Progressive ${ }^{\circledR}$ foi 84,03 . Essa diferença analisada pelo teste de Wilcoxon não foi significante $(\mathrm{p}>0,05)$. Quando se analisou

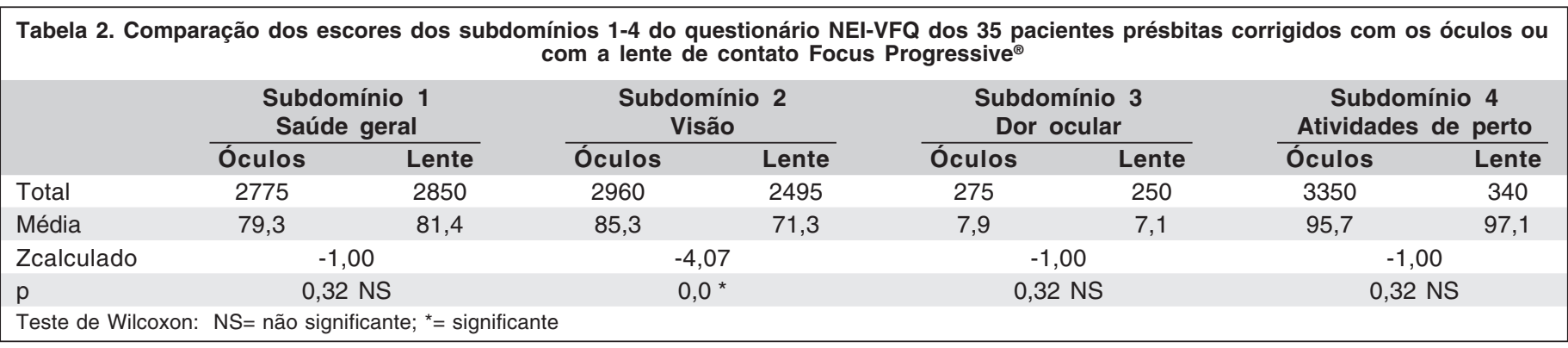

Tabela 3. Comparação dos escores dos subdomínios 5-8 do questionário NEI-VFQ dos 35 pacientes présbitas corrigidos com os óculos ou com a lente de contato Focus Progressive ${ }^{\circledR}$

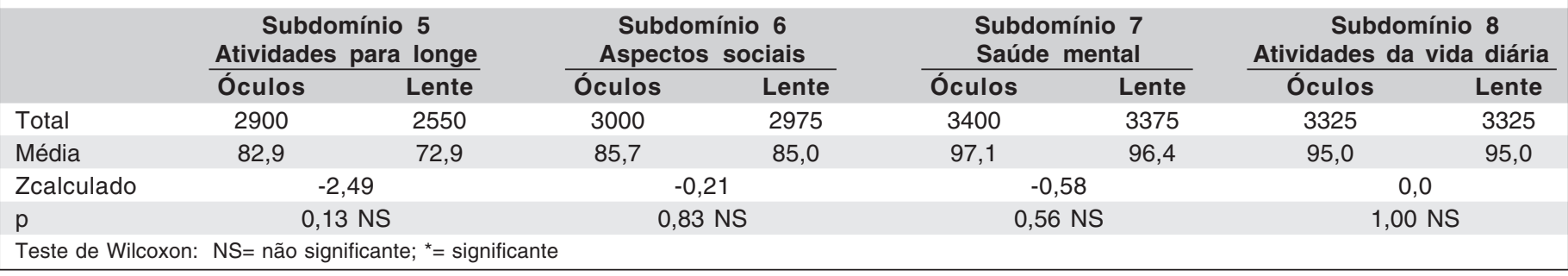

Tabela 4. Comparação dos escores dos subdomínios 9-12 do questionário NEI-VFQ dos 35 pacientes présbitas corrigidos com os óculos ou com a lente de contato Focus Progressive ${ }^{\circledR}$

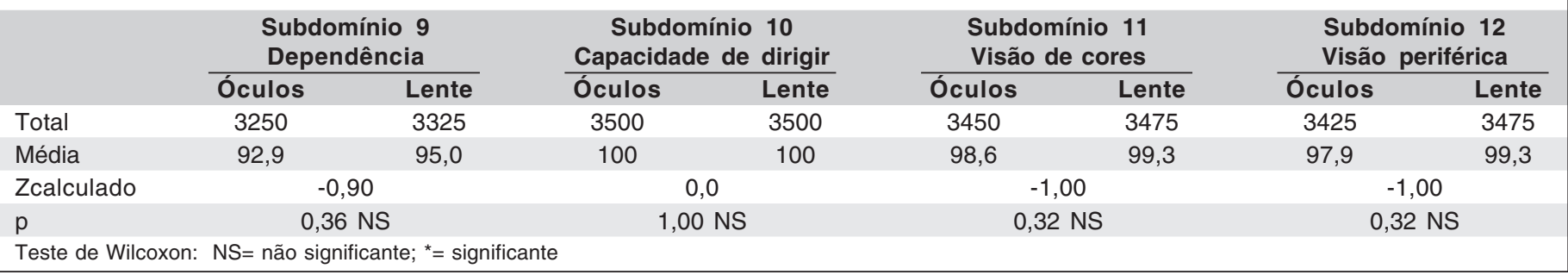


cada subdomínio do questionário neste grupo hipermétrope, observou-se que o subdomínio relativo à visão em geral obteve escore significantemente maior quando a correção com os óculos foi utilizada $(\mathrm{p}<0,001)$ (Tabelas 5, 6, 7).

A média do escore alcançado no questionário NEI-VFQ pela amostra de 10 présbitas com ametropia miópica quando em uso dos óculos foi 85,14 e da lente Focus Progressive ${ }^{\circledR}$ foi 82,63. Essa diferença analisada pelo teste de Wilcoxon não foi signifi- cante ( $\mathrm{p}>0,05)$. Analisando-se cada subdomínio do questionário para esta amostra de míopes, observou-se que o subdomínio relativo à visão em geral obteve escore significantemente maior quando estes usavam os óculos $(\mathrm{p}<0,05)$ (Tabelas 8, 9, 10).

Comparando-se por meio do teste de Wilcoxon, os escores do questionário no grupo hipermétrope com idade inferior à mediana, corrigidos com os óculos $(86,72)$ ou com a lente de contato $(83,61)$, foi observado que os escores do questionário

\begin{tabular}{|c|c|c|c|c|c|c|c|c|}
\hline & \multicolumn{2}{|c|}{$\begin{array}{l}\text { Subdomínio } 1 \\
\text { Saúde geral }\end{array}$} & \multicolumn{2}{|c|}{$\begin{array}{c}\text { Subdomínio } 2 \\
\text { Visão }\end{array}$} & \multicolumn{2}{|c|}{$\begin{array}{l}\text { Subdomínio } 3 \\
\text { Dor ocular }\end{array}$} & \multicolumn{2}{|c|}{$\begin{array}{l}\text { Subdomínio } 4 \\
\text { Atividades de perto }\end{array}$} \\
\hline & Óculos & Lente & Óculos & Lente & Óculos & Lente & Óculos & Lente \\
\hline Total & 1900 & 1975 & 2090 & 1820 & 175 & 150 & 2400 & 2450 \\
\hline Média & 76,0 & 79,0 & 83,6 & 72,8 & 7,0 & 6,0 & 96,0 & 98,0 \\
\hline Zcalculado & \multicolumn{2}{|c|}{$-1,00$} & \multicolumn{2}{|c|}{$-3,28$} & \multicolumn{2}{|c|}{$-1,00$} & \multicolumn{2}{|c|}{$-1,00$} \\
\hline$p$ & \multicolumn{2}{|c|}{0,32 NS } & \multicolumn{2}{|c|}{0,01 * } & \multicolumn{2}{|c|}{0,32 NS } & \multicolumn{2}{|c|}{$0,32 \mathrm{NS}$} \\
\hline
\end{tabular}

\begin{tabular}{|c|c|c|c|c|c|c|c|c|}
\hline & \multicolumn{2}{|c|}{$\begin{array}{l}\text { Subdomínio } 5 \\
\text { Atividades para longe }\end{array}$} & \multicolumn{2}{|c|}{$\begin{array}{l}\text { Subdomínio } 6 \\
\text { Aspectos sociais }\end{array}$} & \multicolumn{2}{|c|}{$\begin{array}{l}\text { Subdomínio } 7 \\
\text { Saúde mental }\end{array}$} & \multicolumn{2}{|c|}{$\begin{aligned} \text { Subdomínio } 8 \\
\text { Atividades da vida diária }\end{aligned}$} \\
\hline & Óculos & Lente & Óculos & Lente & Óculos & Lente & Óculos & Lente \\
\hline Total & 2050 & 1875 & 2125 & 2175 & 2425 & 2425 & 2400 & 2425 \\
\hline Média & 82,0 & 75,0 & 85,0 & 87,0 & 97,0 & 97,0 & 96,0 & 97,0 \\
\hline Zcalculado & \multicolumn{2}{|c|}{$-1,49$} & \multicolumn{2}{|c|}{$-0,451$} & \multicolumn{2}{|c|}{0,0} & \multicolumn{2}{|c|}{$-0,45$} \\
\hline$p$ & \multicolumn{2}{|c|}{$0,14 \mathrm{NS}$} & \multicolumn{2}{|c|}{$0,65 \mathrm{NS}$} & \multicolumn{2}{|c|}{$1,00 \mathrm{NS}$} & \multicolumn{2}{|c|}{$0,65 \mathrm{NS}$} \\
\hline
\end{tabular}

\begin{tabular}{|c|c|c|c|c|c|c|c|c|}
\hline & \multicolumn{2}{|c|}{$\begin{array}{l}\text { Subdomínio } 9 \\
\text { Dependência }\end{array}$} & \multicolumn{2}{|c|}{$\begin{array}{l}\text { Subdomínio } 10 \\
\text { Capacidade de dirigir }\end{array}$} & \multicolumn{2}{|c|}{$\begin{array}{l}\text { Subdomínio } 11 \\
\text { Visão de cores }\end{array}$} & \multicolumn{2}{|c|}{$\begin{array}{l}\text { Subdomínio } 12 \\
\text { Visão periférica }\end{array}$} \\
\hline & Óculos & Lente & Óculos & Lente & Óculos & Lente & Óculos & Lente \\
\hline Total & 2325 & 2375 & 2500 & 2500 & 2450 & 2475 & 2425 & 2475 \\
\hline Média & 93,0 & 95,0 & 100,0 & 100,0 & 98,0 & 99,0 & 97,0 & 99,0 \\
\hline Zcalculado & \multicolumn{2}{|c|}{$-0,70$} & \multicolumn{2}{|c|}{0,0} & \multicolumn{2}{|c|}{$-1,00$} & \multicolumn{2}{|c|}{$-1,00$} \\
\hline$p$ & \multicolumn{2}{|c|}{$0,48 \mathrm{NS}$} & \multicolumn{2}{|c|}{$1,00 \mathrm{NS}$} & \multicolumn{2}{|c|}{$0,32 \mathrm{NS}$} & \multicolumn{2}{|c|}{0,32 NS } \\
\hline
\end{tabular}

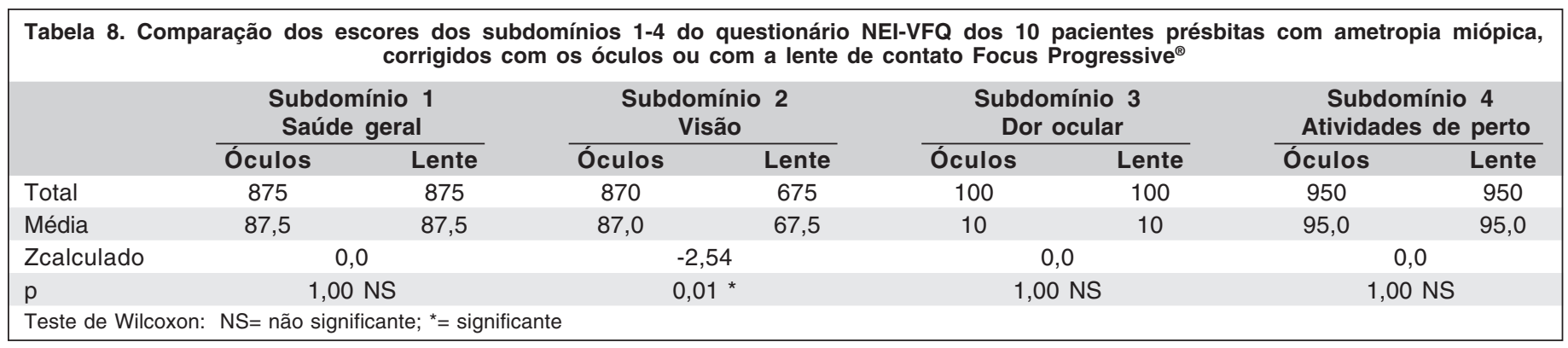




\begin{tabular}{|c|c|c|c|c|c|c|c|c|}
\hline & \multicolumn{2}{|c|}{$\begin{array}{c}\text { Subdomínio } 5 \\
\text { Atividades para longe }\end{array}$} & \multicolumn{2}{|c|}{$\begin{array}{l}\text { Subdomínio } 6 \\
\text { Aspectos sociais }\end{array}$} & \multicolumn{2}{|c|}{$\begin{array}{l}\text { Subdomínio } 7 \\
\text { Saúde mental }\end{array}$} & \multicolumn{2}{|c|}{$\begin{array}{c}\text { Subdomínio } 8 \\
\text { Atividades da vida diária }\end{array}$} \\
\hline & Óculos & Lente & Óculos & Lente & Óculos & Lente & Óculos & Lente \\
\hline Total & 850 & 725 & 875 & 800 & 975 & 950 & 925 & 900 \\
\hline Média & 85,0 & 72,5 & 87,5 & 80,0 & 97,50 & 95,0 & 92,5 & 90,0 \\
\hline Zcalculado & \multicolumn{2}{|c|}{$-2,33$} & \multicolumn{2}{|c|}{$-1,13$} & \multicolumn{2}{|c|}{$-1,00$} & \multicolumn{2}{|c|}{$-1,00$} \\
\hline$p$ & \multicolumn{2}{|c|}{$0,20 \mathrm{NS}$} & \multicolumn{2}{|c|}{$0,26 \mathrm{NS}$} & \multicolumn{2}{|c|}{$0,32 \mathrm{NS}$} & \multicolumn{2}{|c|}{$0,32 \mathrm{NS}$} \\
\hline
\end{tabular}

\begin{tabular}{|c|c|c|c|c|c|c|c|c|}
\hline Total & 925 & 950 & 1000 & 1000 & 1000 & 1000 & 1000 & 1000 \\
\hline Média & 92,5 & 95,0 & 100,0 & 100,0 & 100,0 & 100,0 & 100,0 & 100,0 \\
\hline Zcalculado & \multicolumn{2}{|c|}{$-0,58$} & \multicolumn{2}{|c|}{0,0} & \multicolumn{2}{|c|}{0,0} & \multicolumn{2}{|c|}{0,0} \\
\hline
\end{tabular}

NEI-VFQ foram significantemente maiores quando os óculos eram usados como forma de correção óptica $(\mathrm{p}<0,05)$ (Figura 3).

Entretanto, quando se comparou os escores do questionário de qualidade de vida no grupo présbita com ametropia hipermetrópica, com idade superior à mediana, corrigidos com os óculos ou com a lente de contato, observou-se, por meio do teste de Wilcoxon, que o escore foi significantemente maior quando a lente de contato $(81,36)$ era usada, ao invés dos óculos $(83,75), \mathrm{p}<0,05$ (Figura 4).

Analisando-se os escores do questionário de qualidade de vida no grupo présbita com ametropia miópica com idade igual ou inferior à mediana (47 anos), observou-se que aqueles corrigidos com os óculos apresentaram escores significantemente

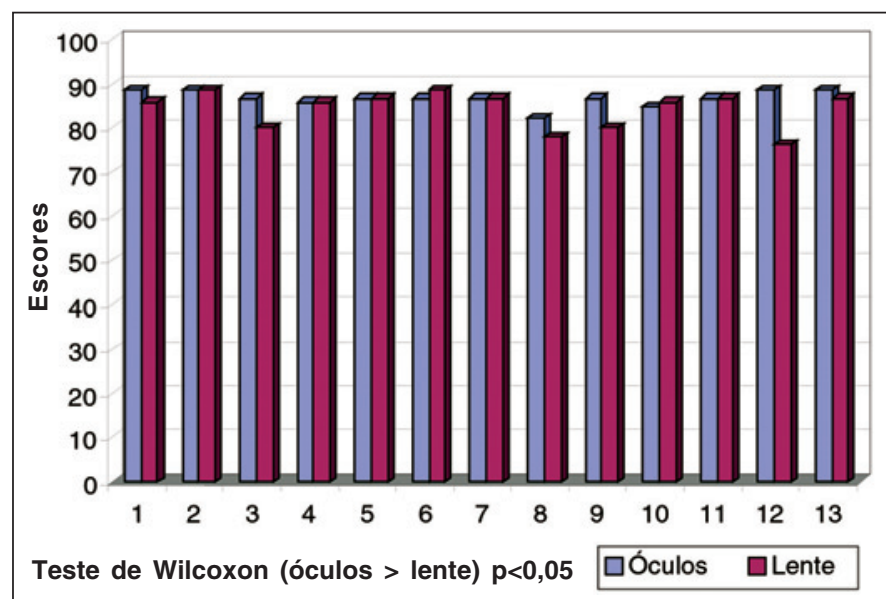

Figura 3 - Média dos escores do questionário NEI VFQ-25 de cada présbita do grupo hipermétrope abaixo da mediana da idade (50 anos) corrigido com os óculos ou com a lente de contato Focus Progressive ${ }^{\circledR}$ melhores $(90,26)$ do que quando em uso da lente de contato $(84,39), p<0,05$ (Figura 5) e naqueles com idade superior à mediana, não houve diferença significante entre os escores do questionário considerando os óculos como forma de correção óptica $(84,10)$ ou a lente de contato $(80,00),(p>0,05)$ (Figura 6).

\section{DISCUSSÃO}

Estudos com diversos desenhos de lente de contato multifocal ou progressiva têm sido realizados e os resultados das medidas de acuidade visual para perto e para longe têm se mostrado inferior aos óculos.

$\mathrm{Na}$ avaliação de 42 pacientes com as lentes Focus Progressive $^{\circledR}$ foi observado que a média da acuidade visual medida

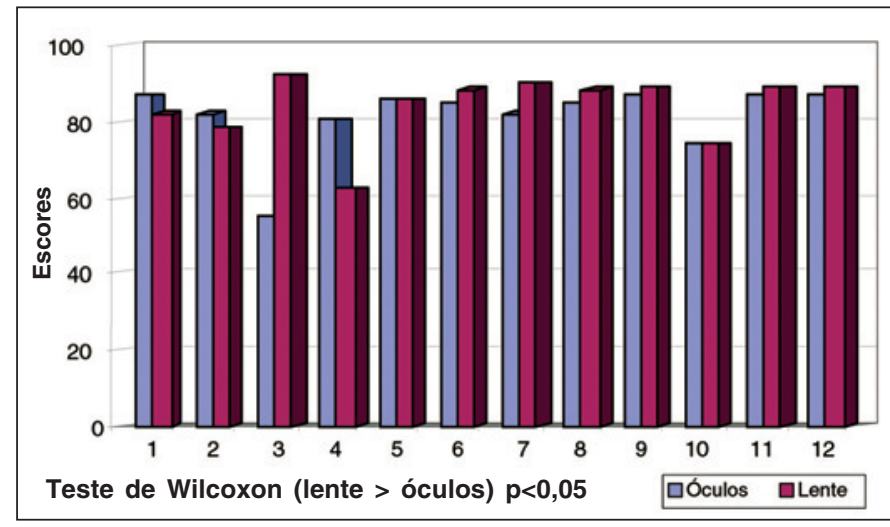

Figura 4 - Média dos escores do questionário NEI VFQ-25 de cada présbita do grupo hipermétrope acima da mediana da idade (50 anos) corrigido com os óculos ou com a lente de contato Focus Progressive ${ }^{\circledR}$ 
com a lente era inferior à medida com óculos, sendo na média meia linha menor quando medida na tabela parametrizada de longe Bailey-Lovie, com alto contraste e em média, meia linha na carta de Jaeger para perto ${ }^{(7)}$.

Resultado semelhante foi encontrado analisando-se o desempenho visual de duas lentes de contato, uma multi zona bifocal (Acuvue bifocal ${ }^{\circledR}$ ) e a outra progressiva de superfície asférica anterior (Focus Progressive ${ }^{\circledR}$ ), onde ambas apresentaram média da acuidade visual logMAR inferior aos óculos em uma linha e uma linha e meia, respectivamente; medidas na tabela de longe de alto contraste e $\mathrm{J} 2$ para perto na carta de Jaeger ${ }^{(9)}$.

Foram avaliados prospectivamente 100 présbitas adaptados com as lentes Unilens ${ }^{\circledR}$ (asférica anterior) por um período de até dois anos e observaram que $84(84 \%)$ atingiam acuidade $\mathrm{J} 1$ na tabela de Jaeger, 14 (14\%) tinham J2 e dois (2\%) J3 ${ }^{(13)}$.

Em uma avaliação subjetiva de 35 pacientes présbitas, por três meses em uso das lentes Focus Progressive ${ }^{\circledR}$, o desempenho para longe recebeu nota 64,3 (de zero a 100) e para perto nota $72,3^{(8)}$.

Em uma análise da qualidade da imagem retiniana em seis olhos usando lentes Acuvue Bifocal ${ }^{\circledR}$, Focus Progressive ${ }^{\circledR}$ e óculos, foi observado que, em ambas as lentes de contato, a qualidade da imagem retiniana era pior do que com os óculos ${ }^{(14)}$.

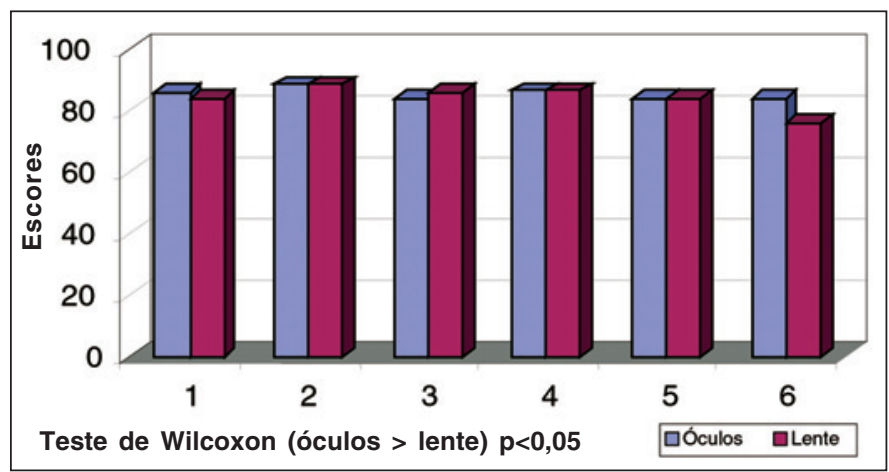

Figura 5 - Média dos escores do questionário NEI VFQ-25 de cada présbita do grupo míope abaixo da mediana da idade (47 anos) corrigido com os óculos ou com a lente de contato Focus Progressive ${ }^{\circledR}$

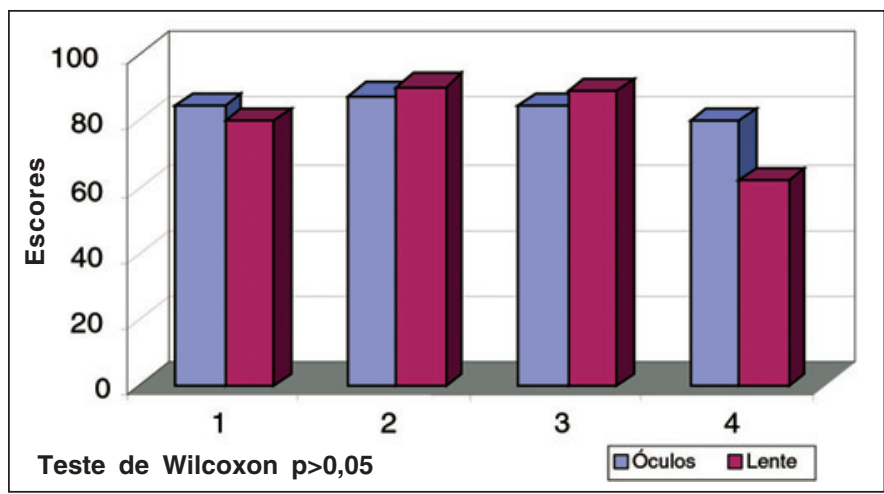

Figura 6 - Média dos escores do questionário NEI VFQ-25 de cada présbita do grupo míope acima da mediana da idade (47 anos) corrigido com os óculos ou com a lente de contato Focus Progressive ${ }^{\circledR}$
Diversos estudos com desenhos diferentes de lente de contato para a presbiopia têm sido desenvolvidos com a finalidade de se mensurar a sensibilidade ao contraste. De todos os desenhos disponíveis comercialmente no mercado, o difrativo é o que mais reduz a sensibilidade ao contraste ${ }^{(6,15-21)}$ e o desenho asférico anterior é o que menos a altera ${ }^{(7,9,13,22-23)}$. Estudos na literatura mostram resultados semelhantes aos do estudo, em lentes de desenho asférico; porém por vezes, com meios diferentes de mensuração da sensibilidade ao contraste. Em um estudo comparativo da lente difrativa Echelon ${ }^{\circledR} \mathrm{com}$ a lente asférica anterior Unilens ${ }^{\circledR} \mathrm{em} 25$ pacientes présbitas, foi notada que a sensibilidade ao contraste medida com a tabela Pelli-Robson a dois metros foi reduzida em média uma linha com as lentes Echelon ${ }^{\circledR}$ e não houve, na média, uma significante redução com as lentes Unilens ${ }^{\circledR(6)}$.

A medida da sensibilidade ao contraste com a lente Focus Progressive ${ }^{\circledR}$ foi testada em 42 pacientes présbitas e foram observadas que as medidas de acuidade visual na tabela BaileyLovie para longe, com baixo contraste, era na média uma linha menor (20/60) do que com óculos (20/50) e para perto, com baixo contraste, também de uma linha pior (J3) do que com óculos $(\mathrm{J} 2)^{(7)}$. Posteriormente, nova avaliação de acuidade visual com a tabela Guillon-Poling em $90 \%$ e $10 \%$ de contraste (10-letter LogMar VA) foi feita em 45 pacientes usuários das lentes Focus Progressive ${ }^{\circledast}$ e foi observada que a acuidade visual média em baixa luminância era equivalente a $0,2(\log M a r)^{(9)}$.

Essa diferença de dados encontrados com os do presente estudo, pode se dever à diferença de métodos usados para avaliar a sensibilidade ao contraste. Vários trabalhos comparando a efetividade de testes de medida de sensibilidade ao contraste têm mostrado que a rede senoidal e o método de Snellen têm resultados diferentes ${ }^{(16-17)}$.

Quanto à avaliação das respostas ao questionário de qualidade de vida, ainda são raros na literatura nacional, estudos envolvendo questionários de qualidade de vida e portadores de vícios de refração ${ }^{(11,24)}$. A maioria deles utiliza questionários que são aplicáveis apenas àquela cultura para a qual foram desenvolvidos ${ }^{(18)}$. A adaptação de questionários utilizados para pacientes com distúrbios visuais permite não somente a exploração dos impactos específicos da dificuldade visual, mas também, comparar diferentes terapêuticas em relação ao risco-benefício ${ }^{(11)}$.

No presente estudo, a avaliação global de todos os présbitas não apresentou diferença significante nas respostas do questionário de qualidade de vida quanto à forma de correção (óculos ou a lente de contato Focus Progressive ${ }^{\circledR}$ ), apesar dos subdomínios relacionados à visão e à visão de longe serem significantemente maiores com os óculos, mostrando que a visão não foi o fator determinante nas respostas deste grupo de pacientes présbitas.

Ao avaliar se as diferentes formas de correção influenciavam nas respostas do questionário, segundo o tipo de ametropia, observou-se que as respostas não diferiram significativamente quanto à forma de correção, independente da ametropia. 
Em uma análise de 100 pacientes com as lentes Unilens (desenho asférico anterior) foi observado que o tipo de ametropia também não influenciou nos dados objetivos e subjetivos de avaliação da lente de contato testada ${ }^{(14)}$

Quando foram analisadas as respostas do questionário NEI VFQ-25 nos grupos hipermétrope acima e abaixo da mediana de idade (50 anos), observou-se que os escores das respostas do questionário de qualidade de vida do grupo com idade abaixo da mediana, foram significantemente maiores quando em uso dos óculos do que das lentes de contato. No grupo hipermétrope com idade acima da mediana, o oposto ocorreu, sendo que os escores quando em uso da lente de contato foram significantemente maiores.

No grupo míope com idade abaixo da mediana (47 anos), os escores das respostas do questionário de qualidade de vida quando em uso dos óculos foram significantemente maiores do que quando em uso da lente de contato e no grupo acima da mediana, não houve diferença significante dos escores, quanto à forma de correção. Esses resultados podem ser explicados pela avaliação severa das desvantagens do sistema de multifocalidade impostos pela lente de contato progressiva, quando a necessidade de correção óptica para perto não é tão grande. Desta forma, os aspectos negativos da lente de contato contam mais intensamente na avaliação subjetiva do paciente ${ }^{(14,23)}$.

Vale ressaltar que o questionário NEI-VFQ tem tido bom resultado em avaliar qualidade de vida em portadores de doenças oculares quando submetidos a tratamentos ${ }^{(24-25)}$. Entretanto, na literatura, temos poucas publicações utilizando o questionário NEI-VFQ em portadores de vícios de refração. Em um estudo de avaliação de qualidade de vida em usuários de óculos, lentes de contato gelatinosa e rígida gás permeável, o NEI-VFQ foi utilizado e os autores concluíram que ele não foi capaz de detectar diferenças de qualidade de vida em formas diferentes de correção de erros refracionais ${ }^{(19)}$. Resultado oposto foi encontrado ao se comparar a qualidade de vida de pacientes antes e depois de procedimentos cirúrgicos ${ }^{(11)}$.

Em 2002 foi desenvolvido um questionário de qualidade de vida para avaliação da qualidade de vida em portadores de erros refrativos (NEI-RQL) com maior consistência interna e reprodutibilidade do que o NEI-VFQ, porém não disponível e validado até o fím deste estudo, na língua portuguesa ${ }^{(25)}$.

Estudos futuros com o NEI-RQL podem ser úteis na avaliação da qualidade de vida de portadores de vícios de refração, quando formas diferentes de correção forem utilizadas, no sentido de se analisar os resultados sob o ponto de vista dos pacientes e não somente pelas medidas de acuidade visual.

\section{CONCLUSÕES}

A função visual com a lente de contato foi significantemente pior do que com os óculos, o tipo de ametropia não influenciou nas respostas do questionário de qualidade de vida quanto à forma de correção (óculos ou lente de contato), mas sim quanto à idade.

\section{ABSTRACT}

Purpose: To compare the visual function and the answers to a questionnaire of quality of life of patients wearing a progressive contact lens or eyeglasses. Methods: The Focus Progressi$\mathrm{ve}^{\circledR}$ contact lens had been fitted in 35 patients with far visual acuity with progressive-addition eyeglasses equal to zero (log MAR) and near J1 (Jaeger). The far and near visual acuities and the measurement of contrast sensitivity were compared when the patients were wearing the eyeglasses or the contact lens and the patients' results of the scores of the quality of life questionnaire (NEI VFQ-25) were analyzed statistically considering the type of ametropy and the age. Results: The far and near visual acuities and the contrast sensitivity measurement were worse with the contact lens than with eyeglasses. The answers to the questionnaire did not differ when were comparing the same patients wearing eyeglasses or contact lens, no matter the type of ametropy. The myopic and the hyperopic subgroups had worse answers to the quality of life questionnaire when corrected with the contact lens than with eyeglasses, both with age equal to or less than their median. Conclusions: The visual function was worse with the contact lens. The type of ametropy did not influence the answers to the quality of life questionnaire considering the optical correction, but age did.

Keywords: Presbyopia; Quality of life; Accommodation, ocular; Contact lenses; Eyeglasses; Visual acuity; Questionnaires; Comparative study

\section{REFERÊNCIAS}

1. Miller D. Optics and refraction a user-friendly guide. Boston: Mosby; 1996

2. Milder B, Rubin ML. Multifocal lenses In: Milder B, Rubin ML. The fine art of prescribing glasses. Florida. Triad Scientific; 1981 p.101-51.

3. Benjamin WJ. Correction with multifocal spectacle lenses. In: Benjamin WJ, editor. Borish's clinical refraction. Philadelphia: W B Saunders; 1998. p.888-927.

4. Sant'Anna NV, Belfort Jr R, Safady MV, Uras R. Aspectos clínicos da adaptação de óculos com lentes progressivas. Arq Bras Oftalmol. 1997;60(1):72-7.

5. Bierly JR, Lim ES, Litteral G, Anderson CW. A quantitative and qualitative assessment of the Solitaire Bifocal Contact Lens. CLAO J. 1995;21(1):20-3.

6. Brenner MB. An objective and subjective comparative analysis of diffractive and front surface aspheric contact lens designs used to correct presbyopia. CLAO J. 1994;20(1):19-22.

7. Fisher K, Bauman E, Schwallie J. Evaluation of two new soft contact lenses for correction of presbyopia: the Focus Progressives multifocal and the Acuvue Bifocal. Int Contact Lens Clin. 2000;26(4):92-103.

8. Alves MR, Holzchuh N, Arieta CE. Experiência clínica com lentes de contato multifocais focus progressive. Rev Bras Oftalmol. 2001;60(8):572-5.

9. Gullon M, Schock SE. Soft contact lens visual performance: a multicenter study. Optom Vis Sci. 1991;68(2):96-103.

10. Ferraz EVAP, Lima CA, Cella W, Arieta CEL. Adaptação de questionário de avaliação da qualidade de vida para aplicação em portadores de catarata. Arq Bras Oftalmol. 2002;65(3):293-8.

11. Belfort R. Avaliação de qualidade visual e de vida de pacientes portadores de ametropias [tese]. São Paulo: Universidade Federal de São Paulo; 2001.

12. Cella DF. Quality of life: concepts and definition. J Pain Symptom Manage. 1994;9(3):186-92.

13. Scott IU, Schein OD, West S, Bandeen-Roche K, Enger C, Folstein MF Functional status and quality of life measurement among ophthalmic patients. Arch Ophthalmol. 1994;112(3):329-35.

14. Shapiro MB, Bredeson DC. A prospective evaluation of Unilens soft multifocal contact lenses in 100 patients. CLAO J. 1994;20(3):189-91. 
15. Pujol J, Gispets J, Arjona M. Optical performance in eyes wearing two multifocal contact lens designs. Ophthalmic Physiol Opt. 2003;23(4):347-60.

16. Bradley A, Hook J, Haeseker J. A comparison of clinical acuity and contrast sensitivity charts: effect of uncorrected myopia. Ophthalmic Physiol Opt. 1991; 11(3):218-26.

17. Lico M, Cidivanes ML, Machado AJ, Velasco e Cruz AA. Medida da função de sensibilidade ao contraste com redes senoidais e E de Snellen em diferentes padrões de perda visual. Arq Bras Oftalmol. 1997;60(2):157-60.

18. Kuyken W, Orley J, Hudelson P, Sartorius N. Quality of life assessment across cultures. Int J Ment Health. 1994;23(2):5-27.

19. Walline JJ, Bailey MD, Zadnik K. Vision-specific quality of life and modes of refractive error correction. Optom Vis Sci. 2000;77(12):648-52.

20. Atebara NH. Image contrast \& the optics of multifocal intraocular lenses [thesis]. Boston: Harvard Medical School; 1991.
21. Bradley A, Abdul Rahman H, Soni PS, Zhang X. Effects of target distance and pupil size on letter contrast sensitivity with simultaneous vision bifocal contact lenses. Optom Vis Sci. 1993;70(6):476-81.

22. Percival SP. Prospective study of a new diffractive bifocal intraocular lens. Eye. 1989;3(Pt 5):571-5.

23. Stein, HA: The management of presbyopia with contact lenses a review CLAO J. 1990;16(1):33-8.

24. Atique D, Goulart DG, Lake JC, Lima FA, Felberg S, Nishiwaki-Dantas MC. Qualidade de vida após transplante penetrante de córnea. Arq Bras Oftalmol. 2002;65(3):351-4.

25. Hays RD, Spritzer KL. National Eye Institute Refractive Error Quality of Life Instrument (NEI-VFQ_42), version 1.0: a manual for scoring and use. Los Angeles, California: Rand Health Sciences; 2002.

\section{QUESTIONÁRIO NEI VFQ 25}

UNIFESP - HSP - Departamento de Oftalmologia - Setor de Psicologia TESTE VFQ - 25 (Questionário de Função Visual)

Este questionário de julho de 1996 foi desenvolvido pela RAND sob o patrocínio do National Eye Institute - National Institute of Health e traduzido ao português no departamento de oftalmologia da Escola Paulista de Medicina - Universidade Federal de São Paulo em novembro de 1996.

* Autorizada reprodução desde que conste o enunciado acima.

\section{Parte 1 - SAÚDE GERAL E VISÃo}

1) Em geral você diria que sua saúde é:
( ) Excelente
( ) Muito boa
( ) Boa
( ) Satisfatória
( ) Ruim

2) No momento, você diria que sua visão, usando óculos ou lentes de contato está:
( ) Excelente
( ) Boa
( ) Satisfatória
( ) Ruim
( ) Muito ruim
( ) Completamente cego

3) Quanto você se preocupa com sua visão?
( ) Não me preocupo
( ) Pouco tempo
( ) Algum tempo
( ) Muito tempo
( ) O tempo todo

4) Quanto de desconforto você tem sentido nos olhos, por exemplo: dor, ardor, queimação ou coceira?
( ) Nada
( ) Suave
( ) Moderada
( ) Severa
( ) Muito severa

\section{Parte 2 - DIFICULDADES NAS ATIVIDADES}

As próximas questões são sobre as dificuldades que você pode ter ao desempenhar certas atividades, com os seus óculos ou lentes de contato se você os usar.

5) Quanta dificuldade você tem na leitura de texto de jornais?

( ) Nenhuma dificuldade

( ) Pouca dificuldade

( ) Moderada dificuldade

( ) Extrema dificuldade

( ) Parei de ler devido à minha visão

( ) Parei de ler por outra razão ou não me interesso por isso
6) Quanta dificuldade você tem em fazer um trabalho que necessite boa visão para perto como cozinhar, costurar ou outros trabaIhos manuais?

( ) Nenhuma dificuldade

( ) Pouca dificuldade

( ) Moderada dificuldade

( ) Extrema dificuldade

( ) Parou de fazer devido à sua visão

( ) Parou de fazer por outra razão ou não se interessa por isso

7) Devido à sua visão quanta dificuldade você tem para encontrar algum objeto numa prateleira repleta de coisas?

( ) Nenhuma dificuldade

( ) Pouca dificuldade

( ) Moderada dificuldade

( ) Extrema dificuldade

( ) Parou de fazer devido à sua visão

( ) Parou de fazer por outra razão ou não se interessa por isso

8) Quanta dificuldade você tem ao ler placas de rua ou nomes de lojas?

( ) Nenhuma dificuldade

( ) Pouca dificuldade

( ) Moderada dificuldade

( ) Extrema dificuldade

( ) Parou de fazer devido à sua visão

( ) Parou de fazer por outra razão ou não se interessa por isso

9) Devido à sua visão, quanta dificuldade você tem para descer escadas ou degraus com pouca luz?

( ) Nenhuma dificuldade

( ) Pouca dificuldade

( ) Moderada dificuldade

( ) Extrema dificuldade

( ) Parou de fazer devido à sua visão

( ) Parou de fazer por outra razão ou não se interessa por isso

10) Devido à sua visão quanta dificuldade você tem em observar coisas que estão ao seu lado enquanto você está caminhando?

( ) Nenhuma dificuldade

( ) Pouca dificuldade

( ) Moderada dificuldade

( ) Extrema dificuldade

( ) Parou de fazer devido à sua visão

( ) Parou de fazer por outra razão ou não se interessa por isso 
11) Devido à sua visão, quanta dificuldade você tem em ver como as pessoas reagem às coisas que você diz?

( ) Nenhuma dificuldade

( ) Pouca dificuldade

( ) Moderada dificuldade

( ) Extrema dificuldade

( ) Parou de fazer devido à sua visão

( ) Parou de fazer por outra razão ou não se interessa por isso

12) Devido à sua visão, quanta dificuldade você tem em escolher e combinar a sua própria roupa?

( ) Nenhuma dificuldade

( ) Pouca dificuldade

( ) Moderada dificuldade

( ) Extrema dificuldade

( ) Parou de fazer devido à sua visão

( ) Parou de fazer por outra razão ou não se interessa por isso

13) Devido à sua visão, quanta dificuldade você tem em visitar pessoas na casa delas, ir a festas ou ir a restaurantes?

( ) Nenhuma dificuldade

( ) Pouca dificuldade

( ) Moderada dificuldade

( ) Extrema dificuldade

( ) Parou de fazer devido à sua visão

( ) Parou de fazer por outra razão ou não se interessa por isso

14) Devido à sua visão, quanta dificuldade você tem em ir ao cinema ou a eventos esportivos?

( ) Nenhuma dificuldade

( ) Pouca dificuldade

( ) Moderada dificuldade

( ) Extrema dificuldade

( ) Parou de fazer devido à sua visão

( ) Parou de fazer por outra razão ou não se interessa por isso

15) Você está dirigindo ao menos de vez em quando?
( ) $\operatorname{Sim}$
( ) Não

15a) Se não: Você nunca dirigiu um carro, ou não tem dirigido?
( ) Nunca dirigi
( ) Dirig

15b) Se você parou de dirigir: Foi essencialmente pela sua visão, por alguma outra razão ou devido a ambas.

( ) Essencialmente pela visão

( ) Essencialmente por outra

( ) Por ambas, visão e outra razão

15c) Se você dirige: Quanta dificuldade você tem para dirigir em locais conhecidos durante o dia?
( ) Nenhuma dificuldade
( ) Pouca dificuldade
( ) Moderada dificuldade
( ) Extrema dificuldade

16) Quanta dificuldade você tem para dirigir à noite?
( ) Nenhuma dificuldade
( ) Pouca dificuldade
( ) Moderada dificuldade
( ) Extrema dificuldade
( ) Parou de fazer devido à sua visão
( ) Parou de fazer por outra razão ou não se interessa por isso

\section{Parte 3 - RESPOSTAS PARA PROBLEMAS DE VISÃO}

As próximas questões são sobre coisas que você pode fazer devido à sua visão. Para as próximas questões indique qual a freqüência mais correta.

17) Você tem menos sucesso do que você gostaria devido à sua visão?

( ) O tempo todo

( ) Na maior parte do tempo

( ) Em alguma parte do tempo

( ) Em pouca parte do tempo

( ) Em nenhuma parte do tempo

18) Você está limitado para executar o seu trabalho ou para fazer outra atividade devido ao seu problema visual?

( ) O tempo todo

( ) Na maior parte do tempo

( ) Em alguma parte do tempo

( ) Em pouca parte do tempo

( ) Em nenhuma parte do tempo

19) Se você sente desconforto nos olhos, por exemplo, ardor, coceira, ou olhos doloridos. Você faz o que gostaria mesmo com desconforto?

( ) O tempo todo

( ) Na maior parte do tempo

( ) Em alguma parte do tempo

( ) Em pouca parte do tempo

( ) Em nenhuma parte do tempo

20) Eu fico em casa a maior parte do tempo devido à minha visão.

( ) Isto é definitivamente verdadeiro

( ) Isto é quase totalmente verdadeiro

( ) Não tenho certeza

( ) Isto é quase totalmente falso

( ) Isto é definitivamente falso

21) Eu me sinto frustrado a maior parte do tempo devido à minha visão.

( ) Isto é definitivamente verdadeiro

( ) Isto é quase totalmente verdadeiro

( ) Não tenho certeza

( ) Isto é quase totalmente falso

( ) Isto é definitivamente falso

22) Eu tenho muito menos controle nas diferentes ações ou tarefas devido à minha visão.

( ) Isto é definitivamente verdadeiro

( ) Isto é quase totalmente verdadeiro

( ) Não tenho certeza

( ) Isto é quase totalmente falso

( ) Isto é definitivamente falso

23) Devido à minha visão eu tenho que confiar mais no que as outras pessoas me contam.

( ) Isto é definitivamente verdadeiro

( ) Isto é quase totalmente verdadeiro

( ) Não tenho certeza

( ) Isto é quase totalmente falso

( ) Isto é definitivamente falso

24) Eu necessito de ajuda dos outros devido à minha visão.

( ) Isto é definitivamente verdadeiro

( ) Isto é quase totalmente verdadeiro

( ) Não tenho certeza

( ) Isto é quase totalmente falso

( ) Isto é definitivamente falso 
25) Devido à minha visão eu me preocupo em fazer coisas que criem problemas para mim ou para os outros.
( ) Isto é definitivamente verdadeiro
( ) Isto é quase totalmente verdadeiro
( ) Não tenho certeza
( ) Isto é quase totalmente falso
( ) Isto é definitivamente falso

\section{Subescala: Visão em geral}

A2) Como você avalia a sua visão com óculos ou lentes de contato numa escala de 0 a 10 , onde 0 significa a pior visão possível e dez significa a melhor visão possível

$\begin{array}{lllllllllll}0 & 1 & 2 & 3 & 4 & 5 & 6 & 7 & 8 & 9 & 10\end{array}$

\section{Subescala: Visão para perto}

A3) Usando óculos, quanta dificuldade você tem para ler letras impressas de lista telefônica, de bula de remédios ou de contratos?

( ) Nenhuma dificuldade

( ) Pouca dificuldade

( ) Moderada dificuldade

( ) Extrema dificuldade

( ) Parou de fazer devido à sua visão

( ) Parou de fazer por outra razão ou não se interessa por isso

A4) Devido à sua visão, quanta dificuldade você tem para conferir as contas que você recebe e ver se elas estão corretas?

( ) Nenhuma dificuldade

( ) Pouca dificuldade

( ) Moderada dificuldade

( ) Extrema dificuldade

( ) Parou de fazer devido à sua visão

( ) Parou de fazer por outra razão ou não se interessa por isso

A5) Devido à sua visão, quanta dificuldade você tem ao fazer coisas como: barbear-se, pentear-se, pintar-se ou maquiar-se?

( ) Nenhuma dificuldade

( ) Pouca dificuldade

( ) Moderada dificuldade

( ) Extrema dificuldade

( ) Parou de fazer devido à sua visão

( ) Parou de fazer por outra razão ou não se interessa por isso

\section{Subescala: Visão para distância}

A6) Devido à sua visão, quanta dificuldade você tem em reconhecer pessoas ao atravessar uma sala?

( ) Nenhuma dificuldade

( ) Pouca dificuldade

( ) Moderada dificuldade

( ) Extrema dificuldade

( ) Parou de fazer devido à sua visão

( ) Parou de fazer por outra razão ou não se interessa por isso

A7) Devido à sua visão, quanta dificuldade você tem ao participar de esportes ou atividades de diversão como: corrida, boliche ou futebol?

( ) Nenhuma dificuldade

( ) Pouca dificuldade

( ) Moderada dificuldade

( ) Extrema dificuldade

( ) Parou de fazer devido à sua visão

( ) Parou de fazer por outra razão ou não se interessa por isso
A8) Devido à sua visão, quanta dificuldade você tem ao assistir programas de televisão?

( ) Nenhuma dificuldade

( ) Pouca dificuldade

( ) Moderada dificuldade

( ) Extrema dificuldade

( ) Parou de fazer isso devido à sua visão

( ) Parou de fazer por outra razão

A9) Devido à sua visão, quanta dificuldade você tem em entreter amigos e familiares em sua casa?

( ) Nenhuma dificuldade

( ) Pouca dificuldade

( ) Moderada dificuldade

( ) Extrema dificuldade

( ) Parou de fazer devido à sua visão

( ) Parou de fazer por outra razão ou não se interessa por isso

\section{Subescala: Dirigir automóveis}

A10) Quanta dificuldade você tem ao dirigir em condições difíceis como: tempo ruim, horários de trânsito pior ou em estradas com tráfego movimentado?

( ) Nenhuma dificuldade

( ) Pouca dificuldade

( ) Moderada dificuldade

( ) Extrema dificuldade

( ) Parou de fazer devido à sua visão

( ) Parou de fazer por outra razão ou não se interessa por isso

\section{Subescala: Limitações}

As próximas questões são sobre coisas que você pode ou não fazer devido à sua visão.

A11) Você necessita de mais ajuda dos outros devido à sua visão?

( ) O tempo todo

( ) Quase o tempo todo

( ) Alguma parte do tempo

( ) Pouca parte do tempo

( ) Nenhuma parte do tempo

A12) Você é limitado nos tipos de coisas que você pode fazer devido à sua visão?

( ) O tempo todo

( ) Quase o tempo todo

( ) Alguma parte do tempo

( ) Pouca parte do tempo

( ) Nenhuma parte do tempo

\section{Subescala: Angústia e dependência}

As próximas questões são sobre como você lida com sua visão.

A13) Eu estou frequentemente irritado devido a minha visão.

( ) Esta afirmação é totalmente verdadeira

( ) Esta afirmação é quase verdadeira

( ) Não tenho certeza

( ) Esta afirmação é quase falsa

( ) Esta afirmação é totalmente falsa

A14) Eu não saio de casa sozinho devido à minha visão

( ) Esta afirmação é totalmente verdadeira

( ) Esta afirmação é quase verdadeira

( ) Não tenho certeza

( ) Esta afirmação é quase falsa

( ) Esta afirmação é totalmente falsa 